\title{
Ultrafast Ptychography with 7500 Frames per Second
}

\author{
Martin Huth $^{1 *}$, Robert Ritz ${ }^{1}$, Colum M. O’Leary ${ }^{2}$, Ian Griffiths ${ }^{2}$, Peter Nellist ${ }^{2}$ and Heike Soltau ${ }^{1}$ \\ 1. PNDetector GmbH, Otto-Hahn-Ring 6, 81739 München, Germany \\ 2. Department of Materials, University of Oxford, 13 Parks Road, Oxford OX13PH, UK \\ * Corresponding author: martin.huth@pndetector.de
}

The field of four-dimensional scanning transmission electron microscopy (4D STEM) imaging, where a focused beam of electrons scans the sample in a two-dimensional (2D) raster pattern, has gained significant interest over the past few years. The possibility of coherent STEM imaging, enabling quantitative phase contrast imaging, extends the range of materials which can be analyzed with STEM down to light elements like carbon. However, beam-sensitive samples, commonly studied in material science or biological applications, may charge or deteriorate during the measurement process requiring optimized measurement conditions. Apart from lowering the electron dose, increasing the readout rate of the detector plays a major role in obtaining high quality and high resolution images.

The pnCCD (S)TEM camera [1] combines the unique features of ultra-fast image acquisition with direct electron detection at single electron sensitivity, ideally suited for low-dose STEM applications. The pnCCD (S)TEM camera can be operated with electron energies ranging from $20 \mathrm{keV}$ to $300 \mathrm{keV}$. By optimizing the operation conditions and timing coordination of the readout ASICs, it was possible to double the speed of the pnCCD (S)TEM camera. In table 1, the list of standard new readout modes is shown.

Here, we present high quality data from 4D STEM measurements recorded with readout speeds of 7500 frames per second while reading out the full sensor area (no windowing), allowing for ultra-low electron doses and reduced irradiation times of the sample. With total scan times of less than $40 \mathrm{~s}$ for a $512 \times 512$ STEM image, the sample drift could be drastically reduced. The ultra-fast readout speed of the pnCCD (S)TEM camera improves high resolution (low-dose) STEM imaging techniques.

The 4D STEM data, presented in Figure 1, were acquired for a 512 x 512 probe position scan using the JEOL 4D Canvas installed on the JEOL ARM200F probe-corrected S/TEM at University of Oxford (HT $=200 \mathrm{kV}, \alpha=31.48 \mathrm{mrad}$, step size $=0.13 \AA$, electron dose $\sim 106 \mathrm{e} \AA^{-2}$ ). A monolayer graphene sample was measured with the regular $4000 \mathrm{fps}$ readout speed using $4 \mathrm{x}$ binning, as well as using the new ultrafast readout scheme running at $7500 \mathrm{fps}$ speed also using 4x binning. Analysis used ptychographic phase reconstruction, which relates the interference between transmitted and diffracted beams in the recorded CBED patterns to the sample-induced electron phase shifts [2]. The method used for reconstruction of the electron phase images, known as single-side-band (SSB) ptychography, involves a Fourier transform of the acquired 4D STEM image, a weak-phase-object approximation and a final inverse Fourier transform. There is no quality difference between standard and accelerated readout modes.

References:

[1] Ryll, H., et al, Journal of Instrumentation 11 (2016)

[2] T. J. Pennycook et al, Ultramicroscopy 151 (2015), p. 160-167.

[3] J. M. Rodenburg et al, Phil. Trans. A 339 (1992), p. 521-553. 
[4] J. M. Rodenburg et al, Ultramicroscopy 48 (1993), p. 304-314.

\begin{tabular}{|c|c|c|c|c|}
\hline & Full frame & Windowing $\mathrm{x} 2$ & Binning $\mathrm{x} 2$ & Binning $\mathrm{x} 4$ \\
\hline rd pnCCD readout & $1000 \mathrm{fps}$ & $2000 \mathrm{fps}$ & $2000 \mathrm{fps}$ & $4000 \mathrm{fps}$ \\
\hline b) new ultrafast readout & $2000 \mathrm{fps}$ & $4000 \mathrm{fps}$ & $4000 \mathrm{fps}$ & $7500 \mathrm{fps}$ \\
\hline
\end{tabular}

Table 1. Comparison of standard (a) and new ultrafast readout modes (b) of the pnCCD (S)TEM camera.

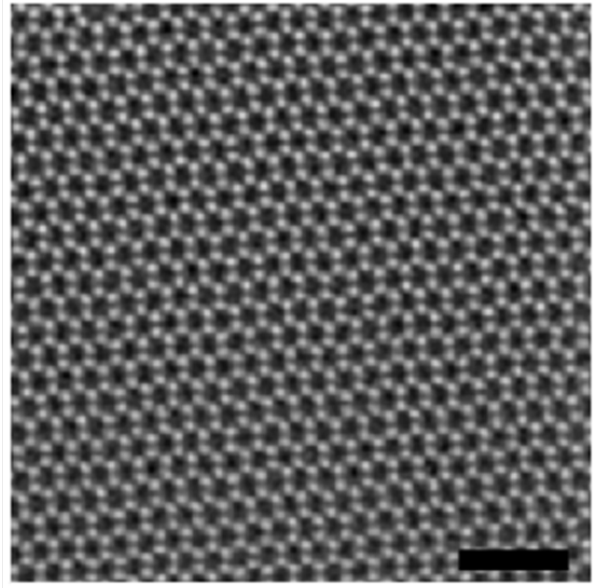

a)

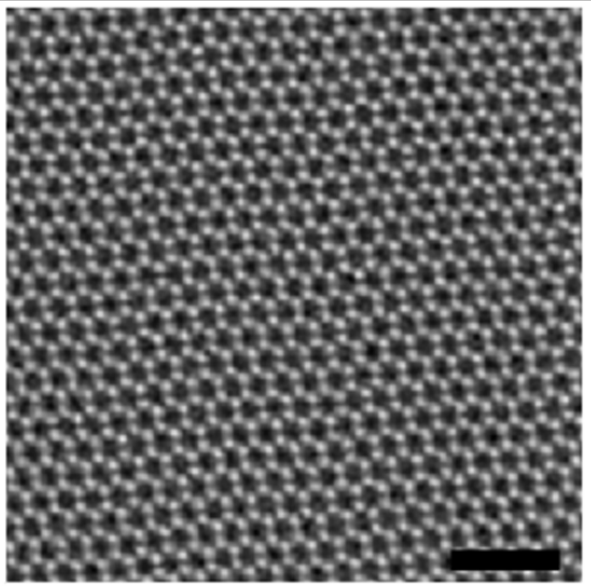

b)

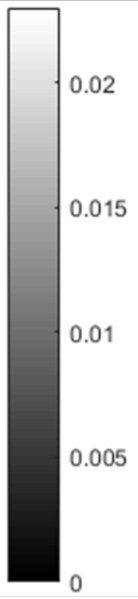

Figure 1. Phase reconstructions using single-side-band electron ptychography (refer to [1]) on 4D
STEM data acquired with the JEOL pnCCD (S)TEM camera (4DCanvas). The data was acquired at (a) $4 \mathrm{kHz}$ and (b) $7.5 \mathrm{kHz}$ frame rates. Scale bar: $1 \mathrm{~nm}$. Image grey scale: phase (radians).

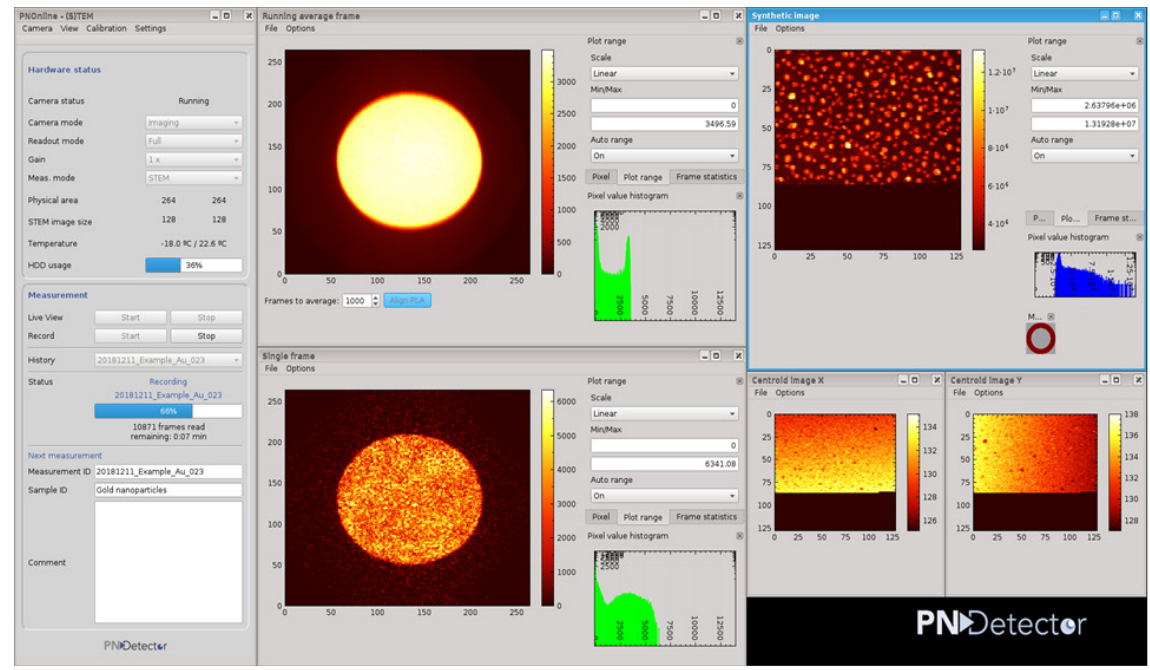

a)

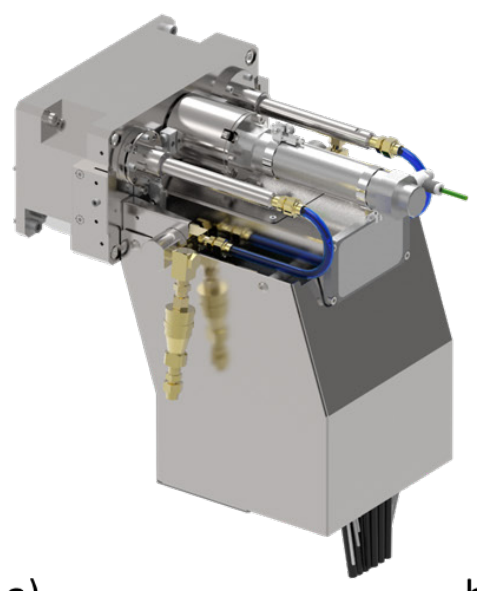

b)

Figure 2. a) Software "PNOnline" for data acquisition and controlling the 4D Canvas camera. b) Picture of the 4D Canvas Detector, which is the JEOL integrated version of the pnCCD (S)TEM camera. 